\title{
Generalized Coefficients for Hopf Cyclic Cohomology ${ }^{\star}$
}

\author{
Mohammad HASSANZADEH, Dan KUCEROVSKY and Bahram RANGIPOUR \\ University of New Brunswick, Fredericton, Canada \\ E-mail:mhassan@uwindsor.ca,dkucerov@unb.ca,bahram@unb.ca
}

Received August 19, 2013, in final form August 22, 2014; Published online September 01, 2014

http://dx.doi.org/10.3842/SIGMA.2014.093

\begin{abstract}
A category of coefficients for Hopf cyclic cohomology is defined. It is shown that this category has two proper subcategories of which the smallest one is the known category of stable anti Yetter-Drinfeld modules. The middle subcategory is comprised of those coefficients which satisfy a generalized SAYD condition depending on both the Hopf algebra and the (co)algebra in question. Some examples are introduced to show that these three categories are different. It is shown that all components of Hopf cyclic cohomology work well with the new coefficients we have defined.
\end{abstract}

Key words: cyclic cohomology; Hopf algebras; noncommutative geometry

2010 Mathematics Subject Classification: 19D55; 16T05; 11M55

Dedicated to Professor Marc A. Rieffel

\section{Introduction}

A suitable class of coefficients for Hopf cyclic cohomology was first introduced in [6, 7]. These coefficients generalize modular pairs in involution discovered by Connes-Moscovici for Hopf cyclic cohomology [4], and are called stable anti Yetter-Drinfeld (SAYD) modules. Hopf cyclic cohomology is defined for a datum consisting of a symmetry, a space, and a space of coefficients. The symmetry is defined by a Hopf algebra, the space is defined by an algebra or a coalgebra on which the Hopf algebra acts or coacts, and the coefficients space is a module and comodule over the Hopf algebra satisfying two conditions defined merely by the Hopf algebra structure. One notes that bialgebra cyclic cohomology introduced in [8] extends the Hopf cyclic cohomology tremendously by relaxing the symmetry to be a bialgebra and the coefficients to be just stable module-comodule. However for computing the latter cohomology one cannot rely on the homological algebra of coalgebras and algebras. What we introduce in this paper is a class of coefficients larger than SAYD modules but still computable in terms of derived category of (co)algebras. We show that the bialgebra cyclic cohomology for the new coefficients coincide with their Hopf cyclic cohomology.

Based on examples, we observe that Hopf cyclic cohomology works well with some coefficients which are not SAYD modules over Hopf algebras. We study these modules and prove that there are at least three noticeable categories of such coefficients. The idea is to show that the coefficients not only depend on the Hopf algebra in question but that they may also be related to the (co)algebra on which the Hopf algebra (co)acts. This observation unties our hands in situations where the Hopf algebra lacks a large class of SAYD modules. Existence of such a Hopf algebra is already shown in [15], where it is proved that any finite-dimensional SAYD module over Connes-Moscovici Hopf algebra is a direct sum of the original modular pair in involution found in [4].

\footnotetext{
${ }^{\star}$ This paper is a contribution to the Special Issue on Noncommutative Geometry and Quantum Groups in honor of Marc A. Rieffel. The full collection is available at http://www.emis.de/journals/SIGMA/Rieffel.html
} 
We cover two cases of Hopf cyclic cohomology in this paper. For an $H$-module coalgebra $C$ we define ${ }_{C} H$-SAYD modules and for an $H$-module algebra $A$ we introduce ${ }_{A} H$-SAYD modules. We show that SAYD modules are both ${ }_{C} H$-SAYD modules and ${ }_{A} H$-SAYD modules for any $A$ and $C$, however we give several examples showing that these inclusions of categories are proper.

Next we show that there are examples of coefficients for $H$-module (co)algebras $(C) A$ which are not even $\left({ }_{C} H\right)_{A} H$-SAYD modules, while the correspondent Hopf cyclic complexes are welldefined. We call these coefficient Hopf cyclic coefficients (HCC). It is not difficult to see that the cup product in Hopf cyclic cohomology works well with these new coefficients.

One notes that after introducing Hopf cyclic cohomology with coefficients in $[6,7]$, there are some generalizations of the theory in $[1,8,11]$. However in all computable examples the authors had to transform their examples into the original Hopf cyclic complexes of some new Hopf algebras. As an important example of such, in [8] the coefficients are module/comodules over bialgebras and do not have to satisfy any YD like condition. However, the author in [8] proves that if these coefficients are SAYD, when the bialgebra is Hopf algebra, then the Hopf cyclic cohomology and bialgebra cyclic cohomoloy coincide. We prove that the same result holds for new coefficients. The proof is a modification of the proof given in [8] for SAYD modules over Hopf algebras.

Hopf cyclic cohomology generalizes Lie algebra cohomology. One of the most useful features of Lie algebra cohomology is the induction/restriction functor which simply allows us to use a subalebra of the Lie algebra in question to calculate the Lie algebra cohomology of the Lie algebra with coefficients in the induced module in terms of the Lie algebra cohomology of the subalgebra with coefficients in the given module. This feature is successfully adopted for Hopf cyclic cohomology in [7]. Thanks to one of the referees some of our examples can be calculated via the induction procedure.

Notations. In this paper we denote a Hopf algebra by $H$ and its counit by $\varepsilon$. We use the Sweedler summation notation $\Delta(h)=h^{(1)} \otimes h^{(2)}$ for the coproduct of a Hopf algebra. Furthermore $\boldsymbol{\nabla}(h)=h_{\langle-1\rangle} \otimes h_{\langle 0\rangle}$ and $\mathbf{\nabla}(h)=h_{\langle 0\rangle} \otimes h_{\langle 1\rangle}$ are used for the left and right coactions of a coalgebra, respectively.

\section{Hopf cyclic cohomology coefficients}

In this section we introduce two generalized versions of SAYD modules for module coalgebras and module algebras. Let a Hopf algebra act on an algebra or a coalgebra, this action being compatible with (co)algebra structure. The main idea here is to show that there are coefficients by which the Hopf cyclic cohomology of the datum is well-defined where these coefficients are not only (co)representations of the Hopf algebras but also represent the algebra or coalgebra in question. Let us recall from [6] that a right-left SAYD module $M$ over a Hopf algebra $H$ is a right module and a left comodule over $H$ such that

$$
\begin{aligned}
& \boldsymbol{\nabla}(m h)=S\left(h^{(3)}\right) m_{\langle-1\rangle} h^{(1)} \otimes m_{\langle 0\rangle} h^{(2)} \quad \text { (AYD condition), } \\
& m_{\langle 0\rangle} m_{\langle-1\rangle}=m \quad \text { (stability condition). }
\end{aligned}
$$

Since bicrossed product Hopf algebras will be used repeatedly in this paper, let us recall this notion here. Let $\mathcal{U}$ and $\mathcal{F}$ be Hopf algebras and furthermore let $\mathcal{U}$ be a right $\mathcal{F}$-comodule coalgebra. One then forms a cocrossed product coalgebra $\mathcal{U} \ltimes \mathcal{F}$ that has $\mathcal{F} \otimes \mathcal{U}$ as underlying vector space and the following coalgebra structure

$$
\Delta(f \ltimes u)=f^{(1)} \ltimes u_{\langle 0\rangle}^{(1)} \otimes f^{(2)} u_{\langle 1\rangle}^{(1)} \ltimes u^{(2)}, \quad \epsilon(f \ltimes u)=\epsilon(f) \epsilon(u) .
$$


Dually if $\mathcal{F}$ is a left $\mathcal{U}$-module algebra, we can endow the underlying vector space $\mathcal{F} \otimes \mathcal{U}$ with an algebra structure, to be denoted by $\mathcal{F} \rtimes \mathcal{U}$, with $1 \rtimes 1$ as its unit and the product given by

$$
(f \rtimes u)(g \rtimes v)=f u^{(1)} g \rtimes u^{(2)} v .
$$

The Hopf algebras $\mathcal{U}$ and $\mathcal{F}$ as above, with the action and coaction as above, form a matched pair of Hopf algebras if they satisfy the following compatibility conditions for any $u \in \mathcal{U}$, and any $f \in \mathcal{F}$

$$
\begin{aligned}
& \epsilon(u f)=\epsilon(u) \epsilon(f), \quad \Delta(u f)=u_{\langle 0\rangle}^{(1)} f^{(1)} \otimes u_{\langle 1\rangle}^{(1)}\left(u^{(2)} f^{(2)}\right), \quad \quad \nabla(1)=1 \otimes 1, \\
& \boldsymbol{\nabla}(u v)=u_{\langle 0\rangle}^{(1)} v_{\langle 0\rangle} \otimes u_{\langle 1\rangle}^{(1)}\left(u^{(2)} v_{\langle 1\rangle}\right), \quad u_{\langle 0\rangle}^{(2)} \otimes\left(u^{(1)} f\right) u_{\langle 1\rangle}^{(2)}=u_{\langle 0\rangle}^{(1)} \otimes u_{\langle 1\rangle}^{(1)}\left(u^{(2)} f\right) .
\end{aligned}
$$

One forms a new Hopf algebra $\mathcal{F} \bowtie \mathcal{U}$, called the bicrossed product of the matched pair $(\mathcal{F}, \mathcal{U})$; it has $\mathcal{F} \ltimes \mathcal{U}$ as underlying coalgebra, $\mathcal{F} \rtimes \mathcal{U}$ as underlying algebra and the antipode is defined by

$$
S(f \bowtie u)=\left(1 \bowtie S\left(u_{\langle 0\rangle}\right)\right)\left(S\left(f u_{\langle 1\rangle}\right) \bowtie 1\right), \quad f \in \mathcal{F}, \quad u \in \mathcal{U}
$$

Throughout the paper all Hopf algebras are assumed to have invertible antipodes.

\subsection{The ${ }_{C} \boldsymbol{H}$-SAYD modules for module coalgebras}

For basics of (co)cyclic modules and their correspondence cyclic (co)homology we refer the reader to [3] and [12]. If $M$ is a right module and left comodule over a Hopf algebra $H$, then for any left $H$-module coalgebra $C$ one defines the following para-cocyclic structure on $C^{*}(C, M):=M \otimes C^{\otimes(n+1)}$

$$
\begin{aligned}
& \partial_{i}(m \otimes \widetilde{c})=m \otimes c_{0} \otimes \cdots \otimes c_{i}^{(1)} \otimes c_{i}^{(2)} \otimes \cdots \otimes c_{n}, \\
& \partial_{n}(m \otimes \widetilde{c})=m_{\langle 0\rangle} \otimes c_{0}^{(2)} \otimes c_{1} \otimes \cdots \otimes c_{n} \otimes m_{\langle-1\rangle} c_{0}^{(1)}, \\
& \sigma_{i}(m \otimes \widetilde{c})=m \otimes c_{0} \otimes \cdots \otimes \varepsilon\left(c_{i}\right) \otimes \cdots \otimes c_{n}, \\
& \tau_{n}(m \otimes \widetilde{c})=m_{\langle 0\rangle} \otimes c_{1} \otimes \cdots \otimes c_{n} \otimes m_{\langle-1\rangle} c_{0},
\end{aligned}
$$

where $\widetilde{c}=c_{0} \otimes \cdots \otimes c_{n}$. In addition, when $M$ is SAYD module over $H$, it is shown in [5] that the above para-cocyclic structure is well-defined on $C_{H}^{n}(C, M):=M \otimes_{H} \otimes C^{\otimes n+1}$. Here $H$ acts on $C^{\otimes n+1}$ diagonally.

However the cyclic structure on $C_{H}^{*}(C, M)$ can be well-defined for some other types of modules $M$ which are not SAYD over $H$.

Definition 2.1. Let $C$ be a left $H$-module coalgebra. A right-left module-comodule $M$ over $H$ is called a $C H$-SAYD module if for any $m \in M, h \in H, c \in C, \tilde{d} \in C^{\otimes \bullet}$ we have

$$
\begin{aligned}
& \left({ }_{C} H \text {-AYD }\right) \quad(m h)_{\langle-1\rangle} c \otimes(m h)_{\langle 0\rangle}=S\left(h^{(3)}\right) m_{\langle-1\rangle} h^{(1)} c \otimes m_{\langle 0\rangle} h^{(2)}, \\
& \left({ }_{C} H \text {-stability) } \quad m_{\langle 0\rangle} \otimes_{H} m_{\langle-1\rangle} \widetilde{d}=m \otimes_{H} \widetilde{d} .\right.
\end{aligned}
$$

Here the action of $H$ on $C^{\otimes \bullet}$ is diagonal. We use the symbol ${ }_{C} H-\mathcal{S} \mathcal{A Y D}$ for the category whose objects are ${ }_{C} H$-SAYD modules and whose morphisms are $H$-module and $H$-comodule morphisms.

Let us introduce some examples of ${ }_{C} H$-SAYD modules which are not necessarily a SAYD module over Hopf algebras. First we generalize the notion of modular pair in involution [4]. 
Lemma 2.2. Let $C$ be an $H$-module coalgebra, $\delta$ a character and $\sigma$ a group-like element for $H$. Let $(\delta, \sigma)$ be a modular pair, i.e. $\delta(\sigma)=1$ and ${ }_{C} H$-in involution, i.e.

$$
S_{\delta}^{2}(h) c=\left(\sigma h \sigma^{-1}\right) c, \quad h \in H, \quad c \in C,
$$

where $S_{\delta}(h)=\delta\left(h^{(1)}\right) S\left(h^{(2)}\right)$. Then ${ }^{\sigma} \mathbb{C}_{\delta}$ is a ${ }_{C} H$-SAYD module via the action and coaction induced by $\delta$ and $\sigma$ respectively.

Proof. One checks that $S_{\delta}^{-1}(h)=S^{-1}\left(h^{(1)}\right) \delta\left(h^{(2)}\right)$. On the other hand we have

$$
\begin{aligned}
& S\left(h^{(3)}\right) \sigma h^{(1)} c \otimes \delta\left(h^{(2)}\right)=S_{\delta}\left(h^{(2)}\right) \sigma h^{(1)} c \otimes 1=S_{\delta}^{2}\left(S_{\delta}^{-1}\left(h^{(2)}\right)\right) \sigma h^{(1)} c \otimes 1 \\
& \quad=\sigma S_{\delta}^{-1}\left(h^{(2)}\right) \sigma^{-1} \sigma h^{(1)} c \otimes 1=\sigma S^{-1}\left(h^{(2)}\right) \delta\left(h^{(3)}\right) h^{(1)} c \otimes 1=\sigma c \otimes \delta(h) .
\end{aligned}
$$

Using the fact that $(\delta, \sigma)$ is a modular pair, the ${ }_{C} H$-stability condition is obvious.

For any $H$-module coalgebra $C$, the following lemma introduce a one-dimensional ${ }_{D} H$-SAYD module for some $H$-module coalgebra $D$.

Lemma 2.3. Let $(\delta, \sigma)$ be a modular pair for the Hopf algebra $H$ and $C$ be a left $H$-module coalgebra. We define the following subspace of $C$

$$
I=\left\{S_{\delta}^{2}(h) c-\left(\sigma h \sigma^{-1}\right) c \mid \text { for all } h \in H, \text { and } c \in C\right\} .
$$

Then $I$ is a coideal of $C$ and $D:=\frac{C}{I}$ is an $H$-module coalgebra. Furthermore ${ }^{\sigma} \mathbb{C}_{\delta}$ is a ${ }_{D} H$-SAYD module.

Proof. Since

$$
S_{\delta}^{2}(h)=\delta\left(h^{(1)}\right) S^{2}\left(h^{(2)}\right) \delta\left(S\left(h^{(3)}\right)\right),
$$

is a coalgebra map, for any $c \in C$ and $h \in H$ we have

$$
\begin{aligned}
\Delta\left(S_{\delta}^{2}(h) c-\sigma h \sigma^{-1} c\right)=S_{\delta}^{2}\left(h^{(1)}\right) c^{(1)} \otimes S_{\delta}^{2}\left(h^{(2)}\right) c^{(2)}-\left(\sigma h^{(1)} \sigma^{-1} c^{(1)}\right) \otimes\left(\sigma h^{(2)} \sigma^{-1} c^{(2)}\right) \\
=S_{\delta}^{2}\left(h^{(1)}\right) c^{(1)} \otimes S_{\delta}^{2}\left(h^{(2)}\right) c^{(2)}-\sigma h^{(1)} \sigma^{-1} c^{(1)} \otimes S_{\delta}^{2}\left(h^{(2)}\right) c^{(2)} \\
\quad-\left(\sigma h^{(1)} \sigma^{-1} c^{(1)}\right) \otimes\left(\sigma h^{(2)} \sigma^{-1} c^{(2)}\right)+\sigma h^{(1)} \sigma^{-1} c^{(1)} \otimes S_{\delta}^{2}\left(h^{(2)}\right) c^{(2)} \\
=\left(S_{\delta}^{2}\left(h^{(1)}\right) c^{(1)}-\sigma h^{(1)} \sigma^{-1} c^{(1)}\right) \otimes S_{\delta}^{2}\left(h^{(2)}\right) c^{(2)} \\
\quad+\left(\sigma h^{(1)} \sigma^{-1} c^{(1)}\right) \otimes\left(S_{\delta}^{2}\left(h^{(2)}\right) c^{(2)}-\left(\sigma h^{(2)} \sigma^{-1} c^{(2)}\right)\right) .
\end{aligned}
$$

This shows $\Delta(I) \subseteq I \otimes C+C \otimes I$. Also we have $\varepsilon(y)=0$ for all $y \in I$. Therefore $I$ is a coideal of $C$ and $D=\frac{C}{I}$ is coalgebra. Now it is enough to show that $I$ is an $H$-module. To do this we show that for all $h, g \in H$ and $c \in C$ we have

$$
h S_{\delta}^{2}(g) c-h \sigma g \sigma^{-1} \in I .
$$

First we observe that

$$
\sigma\left(\sigma^{-1} h \sigma\right) \sigma^{-1}\left(S_{\delta}^{2}(g) c\right)-S_{\delta}^{2}\left(\sigma^{-1} h \sigma\right) S_{\delta}^{2}(g) c \in I .
$$

Since $S_{\delta}^{2}$ is an algebra map, we have

$$
h S_{\delta}^{2}(g) c-\sigma^{-1} S_{\delta}^{2}(h) \sigma S_{\delta}^{2}(g) c \in I .
$$


On the other hand we have

$$
S_{\delta}^{2}\left(\sigma^{-1} h \sigma g\right) c-\sigma\left(\sigma^{-1} h \sigma g\right) \sigma^{-1} \in I .
$$

Therefore

$$
\sigma^{-1} S_{\delta}^{2}(h) \sigma S_{\delta}^{2}(g) c-h \sigma g \sigma^{-1} \in I .
$$

Adding relations (2.3) and (2.4), we obtain (2.2). Therefore $I$ is an $H$-module coalgebra and using definition of $I,(\delta, \sigma)$ is a ${ }_{D} H$-modular pair in involution. Thus by Lemma 2.2 we obtain that ${ }^{\sigma} \mathbb{C}_{\delta}$ is a ${ }_{D} H$-SAYD module.

To exemplify, let us recall that $\mathcal{H}:=\mathcal{H}_{1}^{\text {cop }}$, the co-opposite Hopf algebra of the ConnesMoscovici Hopf algebra in codimension one, [4] is generated by the elements $X, Y$ and $\delta_{k}$, where $k \in \mathbb{N}$, subject to the following relations

$$
[Y, X]=X, \quad\left[Y, \delta_{k}\right]=k \delta_{k}, \quad\left[X, \delta_{k}\right]=\delta_{k+1}, \quad\left[\delta_{k}, \delta_{i}\right]=0, \quad k, i \in \mathbb{N} .
$$

Its coalgebra structure and antipode are defined as follows

$$
\begin{aligned}
& \Delta(Y)=1 \otimes Y+Y \otimes 1, \Delta\left(\delta_{1}\right)=\delta_{1} \otimes 1+1 \otimes \delta_{1}, \\
& \Delta(X)=X \otimes 1+1 \otimes X+Y \otimes \delta_{1}, \\
& \varepsilon(X)=\varepsilon(Y)=\varepsilon\left(\delta_{k}\right)=0, \quad S(X)=-X+Y \delta_{1}, \quad S(Y)=-Y, \quad S\left(\delta_{k}\right)=-\delta_{k} .
\end{aligned}
$$

Let $\mathcal{U}$ be the enveloping algebra of the Lie algebra generated by $X$ and $Y$. Also suppose $\mathcal{F}$ is the co-opposite Hopf algebra of the Hopf algebra generated by all the $\delta_{k}$. It is shown in [13] that $H$ is canonically isomorphic to the bicrossed product Hopf algebra $\mathcal{F} \bowtie \mathcal{U}$ where $\mathcal{U}$ acts on $\mathcal{F}$ via

$$
X \delta_{k}=\delta_{k+1}, \quad Y \delta_{k}=k \delta_{k},
$$

and $\mathcal{F}$ coacts on $\mathcal{U}$ via

$$
\mathbf{\nabla}(X)=X \otimes 1+Y \otimes \delta_{1}, \quad \boldsymbol{\nabla}(Y)=Y \otimes 1 .
$$

A similar decomposition exists for the higher dimensions of this Hopf algebra denoted $\mathcal{H}_{n}^{\text {cop }}$. Its is shown in [15, Theorem 3.16] that there is only one-dimensional SAYD module for the co-opposite Hopf algebra of the Connes-Moscovici Hopf algebra $\mathcal{H}_{1}^{\text {cop }}$, namely ${ }^{1} \mathbb{C}_{\delta}$. Since for $\mathcal{H}_{1}^{\text {cop }}$ we have $S^{2} \neq \mathrm{Id}$, it follows that $\delta \neq \varepsilon$. But in the following example we show that ${ }^{1} \mathbb{C}_{\varepsilon}$ defines a ${ }_{C} \mathcal{H}_{1}^{\text {cop }}$-SAYD module.

Example 2.4. In Lemma 2.3, let $\mathcal{H}=\mathcal{H}_{1}^{\text {cop }}, \delta=\varepsilon, \sigma=1$ and $C=\mathcal{H}$, where the $\mathcal{H}$-module structure is given by the multiplication. The relations

$$
S^{2}(Y)=Y, \quad S^{2}\left(\delta_{k}\right)=\delta_{k}, \quad S^{2}(X)=X-\delta_{1},
$$

show that $I$ is the coideal generated by the elements of the form

$$
\left\{S^{2}\left(X^{n}\right) h-X^{n} h \mid n \in \mathbb{N}\right\} .
$$

Using $S^{2}(X)=X-\delta_{1}, X \delta_{i}=\delta_{i+1}+\delta_{i} X$ and the fact that $S^{2}$ is a morphism of algebras, one obtains the following relation easily by induction

$$
S^{2}\left(X^{n}\right)-X^{n}=\delta_{n}+\sum_{i=1}^{n-1} \delta_{i} h_{n, i},
$$


where $h_{n, i}$ are certain elements in $\mathcal{H}$. This shows that the coideal $I$ is generated by

$$
\left\{\delta_{i} h, h \in \mathcal{H}, i \in \mathbb{N}\right\} .
$$

We define a coalgebra map $\varphi: \mathcal{H} \longrightarrow \mathcal{U}$, given by

$$
f \bowtie u \longmapsto \varepsilon(f) u \text {. }
$$

One checks that $\operatorname{ker}(\varphi)=I$ and therefore $\frac{\mathcal{H}}{I} \cong \mathcal{U}$. As a result the action of $\mathcal{H}$ on $D \cong \mathcal{U}$ which has been defined by multiplication simplifies to

$$
(f \bowtie u)(1 \bowtie v)=\varphi(f \bowtie u v)=\varepsilon(f) u v,
$$

where $f \bowtie u \in \mathcal{H}$ and $1 \bowtie v \in \mathcal{U} \cong D$. Therefore $D=\frac{\mathcal{H}}{I} \cong \mathcal{U}$ is a $\mathcal{H}$-module coalgebra and ${ }^{1} \mathbb{C}_{\varepsilon}$ is a $\mathcal{U} \mathcal{H}$-SAYD module.

\subsection{HCC modules for coalgebras}

Definition 2.5. Let $C$ be a coalgebra which is also an $H$-module but not necessarily modulecoalgebra where the action of $H$ on $C^{\otimes \bullet}$ is diagonal. A module-comodule $M$ over $H$ is called a $(H, C)$-Hopf cyclic coefficients, abbreviated by ${ }_{C} H$-HCC, if the cosimplicial and cyclic operators on $C_{H}^{*}(C, M)$ are well-defined and turn it to a cocyclic module. We use the symbol ${ }_{C} H$ - $\mathcal{H C C}$ for the category whose objects are ${ }_{C} H$-HCC modules and whose morphisms are $H$ module and $H$-comodule morphisms

In the following statement we show that for any ${ }_{C} H$-SAYD module the cocyclic module structure defined in (2.1) is well-defined and therefore we obtain a Hopf cyclic cohomology with these new coefficients:

Proposition 2.6. Let $C$ be an $H$-module coalgebra. Then any ${ }_{C} H$-SAYD module $M$ is $a{ }_{C} H$ HCC.

Proof. Since $C$ is assumed to be an $H$-module coalgebra, all cosimplicial structure operators except the very last coface are well-defined. On the other hand, the last coface is a composition of the first coface and the cyclic operator. Therefore it is enough to show that the cyclic operator is well-defined. Let $\pi: C^{n}(C, M) \rightarrow C_{H}^{n}(C, M)$ be the natural projection then we see that the same proof as in [5] works here

$$
\begin{aligned}
& \pi\left(\tau_{n}\left(m h \otimes_{H} c_{0} \otimes \cdots \otimes c_{n}\right)\right)=(m h)_{\langle 0\rangle} \otimes_{H} c_{1} \otimes \cdots \otimes c_{n} \otimes(m h)_{\langle-1\rangle} c_{0} \\
& \quad=m_{\langle 0\rangle} h^{(2)} \otimes_{H} c_{1} \otimes \cdots \otimes c_{n} \otimes S\left(h^{(3)}\right) m_{\langle-1\rangle} h^{(1)} c_{0} \\
& \quad=m_{\langle 0\rangle} \otimes_{H} h^{(2)} c_{1} \otimes \cdots \otimes h^{(n+1)} c_{n} \otimes h^{(n+2)} S\left(h^{(n+3)}\right) m_{\langle-1\rangle} h^{(1)} c_{0} \\
& \quad=m_{\langle 0\rangle} \otimes_{H} h^{(2)} c_{1} \otimes \cdots \otimes h^{(n+1)} c_{n} \otimes m_{\langle-1\rangle} h^{(1)} c_{0} \\
& \quad=\pi\left(\tau_{n}\left(m \otimes h^{(1)} c_{0} \otimes \cdots \otimes h^{(n+1)} c_{n}\right)\right) .
\end{aligned}
$$

We use the ${ }_{C} H$-AYD module condition in the second equality. To verify the cocyclicity condition, using the ${ }_{C} H$-stability condition one has

$$
\tau_{n}^{n+1}\left(m \otimes_{H} \widetilde{c}\right)=m_{\langle 0\rangle} \otimes_{H} m_{\langle-1\rangle} \widetilde{c}=m \otimes_{H} \widetilde{c} .
$$

Lemma 2.7. Let $C$ be an $H$-module coalgebra. If the action of $H$ on $C$ is cocommutative, i.e.

$$
h^{(1)} c_{1} \otimes h^{(2)} c_{2}=h^{(2)} c_{1} \otimes h^{(1)} c_{2}, \quad h \in H, \quad c_{1}, c_{2} \in C,
$$

then any module $M$ over $H$, with the trivial coaction, defines $a_{C} H$-HCC. 
Proof. Since $C$ is assumed to be an $H$-module coalgebra it suffices to show that $\tau_{n}$ is welldefined. Indeed, let $\pi: C^{n}(C, M) \rightarrow C_{H}^{n}(C, M)$ be the natural projection, then

$$
\begin{aligned}
& \pi\left(\tau_{n}\left(m \otimes h^{(1)} c_{0} \otimes \cdots \otimes h^{(n+1)} c_{n}\right)\right)=m \otimes_{H} h^{(2)} c_{1} \otimes \cdots \otimes h^{(n+1)} c_{n} \otimes h^{(1)} c_{0} \\
& \quad=m \otimes_{H} h^{(1)} c_{1} \otimes \cdots \otimes h^{(n)} c_{n} \otimes h^{(n+1)} c_{0}=m h \otimes_{H} c_{1} \otimes \cdots \otimes c_{n} \otimes c_{0} \\
& \quad=\pi\left(\tau_{n}\left(m h \otimes_{H} c_{0} \otimes \cdots \otimes c_{n}\right)\right) .
\end{aligned}
$$

We use (2.5) in the second equality.

The following statement is a dual result to the previous lemma.

Lemma 2.8. Let $C$ an $H$-module coalgebra. If $H$ acts on $C$ commutatively, i.e.

$$
h g c=g h c, \quad h, g \in H, \quad c \in C,
$$

then any comodule $M$ over $H$, by endowing it with the trivial action, defines a ${ }_{C} H$-SAYD module.

Proof. Using the fact that the action of $H$ on $M$ is via $\varepsilon$ and $H$ acts commutatively on $C$ we have

$$
S\left(h^{(3)}\right) m_{\langle-1\rangle} h^{(1)} c \otimes m_{\langle 0\rangle} h^{(2)}=h^{(1)} S\left(h^{(2)}\right) m_{\langle-1\rangle} c \otimes m_{\langle 0\rangle}=m_{\langle-1\rangle} c \otimes m_{\langle 0\rangle} \varepsilon(h) .
$$

The ${ }_{C} H$-stability condition is obvious by the triviality of the action.

Example 2.9. Let $G$ be a discrete group acting on a set $X$ normally, i.e. $g h x=h g x$ for all $g, h \in G$ and $x \in X$. Then consider the group algebra $H=\mathbb{C} G$ and the obvious coalgebra structure $C_{X}$ on $X$ where each element is considered to be a group-like element. Obviously $C_{X}$ is a module coalgebra over $\mathbb{C} G$ by the action of $G$ on $X$. The action of $H$ on $C_{X}$ is commutative. This introduces a source of ${ }_{C} H$-SAYDs which are not ordinary SAYD modules over $H=\mathbb{C} G$. One notes that all types of SAYD modules $M$ on $\mathbb{C} G$ are known, see [6], which in all cases $G$ graded vector spaces $M=\bigoplus_{g} M_{g}$ where for the (non-trivial) action we have $g m=m$ for all $m \in M_{g}$ and $h m \in M_{h g h^{-1}}$.

We present another example. Since $\mathcal{F}$ is a Hopf subalgebra of $H=\mathcal{F} \bowtie \mathcal{U}$, the Hopf algebra $H$ acts on the coalgebra $C:=H \otimes_{\mathcal{F}} \mathbb{C}$ via left multiplication. It is easy to see that as a coalgebra $C$ is isomorphic to $\mathcal{U}$ via $u \longmapsto(1 \bowtie u) \otimes_{\mathcal{F}}$. Via this identification, the action of $H$ on $C$ is as follows. Let $h=f \bowtie u \in H$, and $v \in C=\mathcal{U}$. Then

$$
\begin{aligned}
(f \bowtie u) v & =\left((f \bowtie u)(1 \bowtie v) \otimes_{\mathcal{F}} 1\right) \\
& =(f \bowtie u v) \otimes_{\mathcal{F}} 1=\left[\left(1 \bowtie u^{(1)} v^{(1)}\right)\left(S\left(\left(u^{(2)} v^{(2)}\right)\right) f \bowtie 1\right)\right] \otimes_{\mathcal{F}} 1 \\
& =\left(1 \bowtie u^{(1)} v^{(1)}\right) \otimes_{\mathcal{F}} \varepsilon\left(S\left(\left(u^{(2)} v^{(2)}\right)\right) f\right)=(1 \bowtie u v) \otimes_{\mathcal{F}} \varepsilon(f)=\varepsilon(f) u v .
\end{aligned}
$$

One easily checks that for any bicrossed product Hopf algebra $\mathcal{F} \bowtie \mathcal{U}$, the Hopf algebra $\mathcal{U}$ is an $H$-module coalgebra via this action.

Example 2.10. Consider any bicrossed product Hopf algebra $H=\mathcal{F} \bowtie \mathcal{U}$ where $\mathcal{U}$ is a cocommutative Hopf algebra. We have seen that $C=\mathcal{U}$ is an $H$-module coalgebra by the following action

$$
(f \bowtie u) v=\varepsilon(f) u v .
$$

Since $\mathcal{U}$ is cocommutative, it is obvious that $\mathcal{H}$ acts on $C$ cocommutatively. Therefore any comodule $M$ over $H$ with the trivial action defines a $\mathcal{U} H$-SAYD module. 
Example 2.11. Consider any bicrossed product Hopf algebra $H=\mathcal{F} \bowtie \mathcal{U}$ where $\mathcal{U}$ is a cocommutative Hopf algebra. Consider the module coalgebra $\mathcal{U}$ with the action defined in (2.6). We show that $H$ acts on $\mathcal{U}$ cocommutatively. First let us recall that

$$
\Delta(f \bowtie u)=f^{(1)} \bowtie u_{\langle 0\rangle}^{(1)} \otimes f^{(2)} u_{\langle 1\rangle}^{(1)} \bowtie u_{\langle 2\rangle} .
$$

Using the cocommutativity of $\mathcal{U}$, for $h:=f \bowtie u$ we get

$$
\begin{aligned}
& h^{(1)} v^{1} \otimes h^{(2)} v^{2}=\varepsilon\left(f^{(1)}\right) u_{\langle 0\rangle}^{(1)} v^{1} \otimes \varepsilon\left(f^{(2)} u_{\langle 1\rangle}^{(1)}\right) u^{(2)} v^{2}, \\
& \varepsilon(f) u^{(1)} v^{1} \otimes u^{(2)} v^{2}=\varepsilon(f) u^{(2)} v^{2} \otimes u^{(1)} v^{1}=h^{(2)} v^{1} \otimes h^{(1)} v^{2} .
\end{aligned}
$$

Therefore any module $M$ over $H$, providing it with the trivial coaction of $H$, defines a $\mathcal{U} H$-HCC. As an example the co-opposite Hopf algebra of Connes-Moscovici Hopf algebra $\mathcal{H}_{1}^{\text {cop }} \cong \mathcal{F} \bowtie \mathcal{U}$ acts cocommutatively on $\mathcal{U}$.

Here we introduce an example of ${ }_{C} H$-HHC which is not a ${ }_{C} H$-SAYD module. We have shown that if $H$ acts on $C$ cocommutatively then any module $M$ defines an ${ }_{C} H$-HHC. Indeed in this example, we introduce a triple $(H, C, M)$ such that $H$ acts on $C$ cocommutatively but $M$ is not a ${ }_{C} H$-SAYD module.

Example 2.12. Consider $\mathcal{H}=\mathcal{H}_{1}^{\text {cop }} \cong \mathcal{F} \bowtie \mathcal{U}$. We have seen that $C=\mathcal{U}$ is a $\mathcal{H}$-module coalgebra by the action $(f \bowtie u) v=\varepsilon(f) u v$ and that this action is a cocommutative action by Example 2.11. Now let $M=\mathcal{H}$ be a right $\mathcal{H}$-module via multiplication and left $\mathcal{H}$-comodule by trivial coaction. Since the action is cocommutative, it is a $\mathcal{U} \mathcal{H}$-HCC module. We show that $M$ is not a $C \mathcal{H}$-SAYD. Let $f \bowtie u:=\delta_{1} \otimes X \in \mathcal{H}, c:=X \in \mathcal{U}$, and $m:=1 \bowtie 1 \in M$. First we notice that

$$
\begin{aligned}
& (f \bowtie u)^{(1)} \otimes(f \bowtie u)^{(2)} \otimes(f \bowtie u)^{(3)} \\
& \quad=f^{(1)} \bowtie u_{\langle 0\rangle}^{(1)} \otimes f^{(2)} u_{\langle 1\rangle}^{(1)} \bowtie u_{\langle 0\rangle}^{(2)} \otimes f^{(3)} u_{\langle 2\rangle}^{(1)} u_{\langle 1\rangle}^{(2)} \bowtie u^{(3)} .
\end{aligned}
$$

After substituting into the AYD condition we observe that

$$
\begin{aligned}
S((f & \left.\bowtie u)^{(3)}\right)(f \bowtie u)^{(1)} c \otimes m(f \bowtie u)^{(2)} \\
& =S\left(f^{(3)} u_{\langle 2\rangle}^{(1)} u_{\langle 1\rangle}^{(2)} \bowtie u^{(3)}\right)\left(f^{(1)} \bowtie u_{\langle 0\rangle}^{(1)}\right) c \otimes m f^{(2)} u_{\langle 1\rangle}^{(1)} \bowtie u_{\langle 0\rangle}^{(2)} \\
& =S\left(f^{(2)} u_{\langle 2\rangle}^{(1)} u_{\langle 1\rangle}^{(2)} \bowtie u^{(3)}\right) u_{\langle 0\rangle}^{(1)} c \otimes m f^{(1)} u_{\langle 1\rangle}^{(1)} \bowtie u_{\langle 0\rangle}^{(2)} \\
& =\left(1 \bowtie S\left(u_{\langle 0\rangle}^{(3)}\right)\right)\left(S\left(f^{(2)} u_{\langle 2\rangle}^{(1)} u_{\langle 1\rangle}^{(2)} u_{\langle 1\rangle}^{(3)}\right) \bowtie 1\right) u_{\langle 0\rangle}^{(1)} c \otimes m f^{(1)} u_{\langle 1\rangle}^{(1)} \bowtie u_{\langle 0\rangle}^{(2)} \\
& =S\left(X^{(3)}\right) X_{\langle 0\rangle}^{(1)} X \otimes(1 \bowtie 1)\left(\delta_{1} X_{\langle 1\rangle}^{(1)} \bowtie X^{(2)}\right) \\
& =X_{\langle 0\rangle} X \otimes\left(\delta_{1} X_{\langle 1\rangle} \bowtie 1\right)+X \otimes\left(\delta_{1} \bowtie X\right)+S(X) X \otimes\left(\delta_{1} \bowtie 1\right) \\
& =X^{2} \otimes\left(\delta_{1} \bowtie 1\right)+Y X \otimes\left(\delta_{1}^{2} \bowtie 1\right)+X \otimes\left(\delta_{1} \bowtie X\right)-X^{2} \otimes\left(\delta_{1} \bowtie 1\right) \\
& =X \otimes\left(\delta_{1} \bowtie X\right)+Y X \otimes\left(\delta_{1}^{2} \bowtie 1\right) \neq X \otimes\left(\delta_{1} \bowtie X\right)=c \otimes m h .
\end{aligned}
$$

\subsection{Relation to Kaygun's cyclic cohomology of bialgebras}

In this subsection we first recall the bialgebra cyclic cohomology developed by Kaygun in [8] and then show that for any ${ }_{C} H$-SAYD module the result of these two cohomology coincide.

Let $H$ be a bialgebra, $C$ be an $H$-module coalgebra, $M$ be a right module and left comodule over $H$.

We let $H$ act on $C^{n}(C, M)$ from left by

$$
L_{g}\left(m \otimes c_{0} \otimes \cdots \otimes c_{n}\right)=m S\left(g^{(1)}\right) \otimes g^{(2)} c_{0} \otimes \cdots \otimes g^{(n+2)} c_{n},
$$


and defines the following subspace of $C^{n}(H, M)$

$$
W^{n}(C, M):=\left\{\left[L_{g}, \tau^{i}\right]\left(C^{n}(H, M)\right) \mid g \in H, i \in \mathbb{Z}^{+}\right\} .
$$

Here $\left[L_{g}, \tau\right]:=L_{g} \tau-\tau L_{g}$.

Let us see that $W$ is stable under cyclic structure of $C^{*}(C, M)$. Indeed for $\tau$ we simply see that

$$
\tau\left[L_{g}, \tau^{i}\right]=\left[\tau, L_{g}\right] \tau^{i}+\left[L_{g}, \tau^{i+1}\right] .
$$

For $\partial_{m}$, where $0 \leq m \leq n$, we use the identity $\partial_{m} \tau=\tau \partial_{m+1}$ and the fact that $\partial_{m} L_{g}=L_{g} \partial_{m}$ to see that

$$
\partial_{m}\left[L_{g}, \tau^{i}\right]=\left[L_{g}, \tau\right] \partial_{m+1}
$$

For $\partial_{n+1}=\tau \partial_{0}$ we have

$$
\partial_{0}\left[L_{g}, \tau^{i}\right]=\tau\left[L_{g}, \tau^{i}\right] \partial_{1}=\left[\tau, L_{g}\right] \tau^{i} \partial_{1}+\left[L_{g}, \tau^{i+1}\right] \partial_{1} .
$$

Using the facts that $L_{g} \sigma_{j}=\sigma_{j} L_{g}$ and $\tau \sigma_{j}=\sigma_{j-1} \tau$, we see that for $1 \leq j \leq n$, we have

$$
\sigma_{j}\left[L_{g}, \tau^{i}\right]=\left[L_{g}, \tau^{i}\right] \sigma_{j-1} .
$$

Finally for $\sigma_{0}=\sigma_{n} \tau$ we obtain

$$
\sigma_{0}\left[L_{g}, \tau^{i}\right]=\sigma_{n} \tau\left[L_{g}, \tau^{i}\right]=\left[\tau, L_{g}\right] \sigma_{n-1} \tau^{i}+\left[L_{g}, \tau^{i+1}\right] \sigma_{n-1} .
$$

So $\frac{C^{*}(C, M)}{W^{*}(C, M)}$ is a paracocyclic $H$-module. This paracocyclic module is denoted by $\mathbb{P C M}^{*}(C, M)$ in [8]. On the other hand if $M$ is stable then

$$
\begin{aligned}
& \tau^{n+1}\left(m \otimes c_{0} \otimes \cdots \otimes c_{n}\right)=m_{\langle 0\rangle} \otimes m_{\langle-n-1\rangle} c_{0} \otimes \cdots \otimes m_{\langle-1\rangle} c_{n} \\
& \quad=m_{\langle 0\rangle} m_{\langle-n-3\rangle} S\left(m_{\langle-n-2\rangle}\right) \otimes m_{\langle-n-1\rangle} c_{0} \otimes \cdots \otimes m_{\langle-1\rangle} c_{n} \\
& \quad=L_{m_{\langle-2\rangle}}\left(m_{\langle 0\rangle} m_{\langle-1\rangle} \otimes c_{0} \otimes \cdots \otimes c_{n}\right)=L_{m_{\langle-1\rangle}}\left(m_{\langle 0\rangle} \otimes c_{0} \otimes \cdots \otimes c_{n}\right),
\end{aligned}
$$

which proves that the paracocyclic module $\mathbb{C M}^{*}(C, M):=\mathbb{C} \otimes_{H} \mathbb{P C M}^{*}(C, M)$ is in fact a cocyclic module.

Proposition 2.13. Let $H$ be a Hopf algebra, $C$ be an $H$-module coalgebra, and $M$ be $a{ }_{C} H$ SAYD module. Then $W \subseteq \operatorname{ker} \pi$, where $\pi: C^{n}(C, M) \rightarrow C_{H}^{n}(C, M)$ is the canonical projection.

Proof. Let us fix $m \otimes c_{0} \otimes \cdots \otimes c_{n} \in C^{n}(C, M)$. Using the ${ }_{C} H$-SAYD property of $M$, we see that

$$
\begin{aligned}
& \tau L_{g}\left(m \otimes c_{0} \otimes \cdots \otimes c_{n}\right)=\tau\left(m S\left(g^{(1)}\right) \otimes g^{(2)} c_{0} \otimes \cdots \otimes g^{(n+2)} c_{n}\right) \\
& \quad=\left(m S\left(g^{(1)}\right)\right)_{\langle 0\rangle} \otimes g^{(3)} c_{1} \otimes \cdots \otimes g^{(n+2)} c_{n} \otimes\left(m S\left(g^{(1)}\right)\right)_{\langle-1\rangle} g^{(2)} c_{0} \\
& \quad=m_{\langle 0\rangle} S\left(g^{(2)}\right) \otimes g^{(5)} c_{1} \otimes \cdots \otimes g^{(n+2)} c_{n} \otimes S^{2}\left(g^{(1)}\right) m_{\langle-1\rangle} S\left(g^{(3)}\right) g^{(4)} c_{0} \\
& \quad=m_{\langle 0\rangle} S\left(g^{(2)}\right) \otimes g^{(3)} c_{1} \otimes \cdots \otimes g^{(n+2)} c_{n} \otimes S^{2}\left(g^{(1)}\right) m_{\langle-1\rangle} c_{0} .
\end{aligned}
$$

On the other hand we have

$$
\begin{aligned}
& L_{g} \tau\left(m \otimes c_{0} \otimes \cdots \otimes c_{n}\right)=L_{g}\left(m_{\langle 0\rangle} \otimes c_{1} \otimes \cdots \otimes c_{n} \otimes m_{\langle-1\rangle} c_{0}\right) \\
& \quad=m_{\langle 0\rangle} S\left(g^{(1)}\right) \otimes g^{(2)} c_{1} \otimes \cdots \otimes g^{(n+1)} c_{n} \otimes g^{(n+2)} m_{\langle-1\rangle} c_{0} .
\end{aligned}
$$

So $\pi\left(\tau L_{g}\left(m \otimes c_{0} \otimes \cdots \otimes c_{n}\right)\right)=\varepsilon(g) \tau\left(m \otimes c_{0} \otimes \cdots \otimes c_{n}\right)=\pi\left(L_{g} \tau\left(m \otimes c_{0} \otimes \cdots \otimes c_{n}\right)\right)$. 
To finish the proof one uses the facts that, by Proposition 2.6, $\tau$ on $C_{H}(C, M)$ is well-defined, and that $\left[L_{g},-\right]$ is a derivation. Indeed,

$$
\begin{aligned}
& \pi\left(\left[L_{g}, \tau^{i}\right]\left(m \otimes c_{0} \otimes \cdots \otimes c_{n}\right)\right) \\
& \quad=\pi\left(\left[L_{g}, \tau\right] \tau^{i-1}\left(m \otimes c_{0} \otimes \cdots \otimes c_{n}\right)\right)+\tau^{i-1} \pi\left(\left[L_{g}, \tau\right]\left(m \otimes c_{0} \otimes \cdots \otimes c_{n}\right)\right)=0 .
\end{aligned}
$$

Finally we prove the main result of this subsection.

Proposition 2.14. Let $H$ be a Hopf algebra, $C$ be an $H$-module coalgebra, and $M$ be $a{ }_{C} H$ SAYD module. Then $\mathbb{C M}^{*}(C, M)$ and $C_{H}^{*}(C, M)$ are isomorphic as cocyclic modules.

Proof. The proof is similar to the case of of the SAYD modules [8]. By Proposition 2.13 we have the $H$-linear map between paracocyclic modules

$$
\pi: \mathbb{P C M}^{n}(C, M) \longrightarrow C_{H}^{n}(H, M) \text {. }
$$

Here we assume that the action of $H$ on $C_{H}^{n}(C, M)$ is trivial. It is easily seen that

$$
\partial\left(L_{g}\left(m \otimes c_{0} \otimes \cdots \otimes c_{n}\right)\right)=\varepsilon(g) \pi\left(m \otimes c_{0} \otimes \cdots \otimes c_{n}\right) .
$$

So $\pi$ induces a map of cocyclic modules

$$
\Pi: \mathbb{C M}^{n}(C, M) \longrightarrow C_{H}^{n}(C, M) .
$$

On the other hand since $W^{*}$ is a para-cocyclic submodule of $C^{*}(C, M)$ we have projections of para-cocyclic modules.

$$
\pi^{\prime}: C^{n}(C, M) \longrightarrow \mathbb{C M}^{n}(C, M) .
$$

Let us check that $\pi^{\prime}$ is balanced over $H$. In the following we use $[x]$ as the class of $x \in C^{n}(C, M)$ in $\mathbb{P C M}^{n}(C, M)$ and $\mathbf{1} \in \mathbb{C}$ as the generator of $\mathbb{C}$ as trivial $H$-module.

$$
\begin{aligned}
& \pi^{\prime}\left(m g \otimes c_{0} \otimes \cdots \otimes c_{n}\right)=\mathbf{1} \otimes_{H}\left[m g \otimes c_{0} \otimes \cdots \otimes c_{n}\right]=\mathbf{1} \otimes_{H}\left[m g^{(1)} \varepsilon\left(g^{(2)}\right) \otimes c_{0} \otimes \cdots \otimes c_{n}\right] \\
& \quad=\mathbf{1} \otimes_{H}\left[m g^{(1)} S\left(h^{(2)}\right) \otimes h^{(3)} c_{0} \otimes \cdots \otimes h^{(n+3)} c_{n}\right] \\
& \quad=\mathbf{1} \otimes_{H}\left[m \otimes g^{(1)} c_{0} \otimes \cdots \otimes g^{(n+1)} c_{n}\right]=\pi^{\prime}\left(m \otimes g^{(1)} c_{0} \otimes \cdots \otimes g^{(n+1)} c_{n}\right) .
\end{aligned}
$$

So $\pi^{\prime}$ induces a map of cocyclic modules

$$
\Pi^{\prime}: C_{H}^{n}(C, M) \longrightarrow \mathbb{C M}^{n}(C, M) .
$$

It is obvious that $\Pi$ and $\Pi^{\prime}$ are inverse to each other.

\subsection{The ${ }_{A} H$-SAYD and HCC modules for module algebras}

Let $A$ be a left $H$-module algebra and $M$ be a right-left SAYD module over $H$. We notice that $M \otimes A^{\otimes(n+1)}$ is a right $H$-module via $(m \otimes \widetilde{a}) h:=m h^{(1)} \otimes S\left(h^{(2)}\right) \widetilde{a}$ and the right $H$ module structure of the ground field $\mathbb{C}$ is given by $r h:=\varepsilon(h) r$ for all $r \in \mathbb{C}$ and $h \in H$. It is shown that the following cocyclic structure on the space of right $H$-linear homomorphisms $C_{H}^{n}(A, M):=\operatorname{Hom}_{H}\left(M \otimes A^{\otimes(n+1)}, \mathbb{C}\right)$ is well-defined [6]

$$
\begin{aligned}
& \left(d_{i} f\right)(m \otimes \widetilde{a})=f\left(m \otimes a_{0} \otimes \cdots \otimes a_{i} a_{i+1} \otimes \cdots \otimes a_{n}\right), \quad 0 \leq i<n, \\
& \left(d_{n} f\right)(m \otimes \widetilde{a})=f\left(m_{\langle 0\rangle} \otimes\left(S^{-1}\left(m_{\langle-1\rangle}\right) a_{n}\right) a_{0} \otimes a_{1} \otimes \cdots \otimes a_{n-1}\right), \\
& \left(s_{n} f\right)(m \otimes \widetilde{a})=f\left(m \otimes a_{0} \otimes \cdots \otimes a_{i} \otimes 1 \otimes \cdots \otimes a_{n}\right), \quad 0 \leq i \leq n, \\
& \left(t_{n} f\right)(m \otimes \widetilde{a})=f\left(m_{\langle 0\rangle} \otimes S^{-1}\left(m_{\langle-1\rangle}\right) a_{n} \otimes a_{0} \otimes \cdots \otimes a_{n-1}\right) .
\end{aligned}
$$

where $\widetilde{a}=a_{0} \otimes \cdots \otimes a_{n}$. 
Definition 2.15. Let $A$ be a left $H$-module algebra. A right-left module-comodule $M$ over $H$ is called an ${ }_{A} H$-SAYD module if for all $m \in M, h \in H, a \in A$ and $\varphi \in C_{H}^{*}(A, M)$

i) $S^{-1}\left((m h)_{\langle-1\rangle}\right) a \otimes(m h)_{\langle 0\rangle}=S^{-1}\left(m_{\langle-1\rangle} h^{(1)}\right) h^{(3)} a \otimes m_{\langle 0\rangle} h^{(2)}$,

ii) $\varphi\left(m_{\langle 0\rangle} \otimes S^{-1}\left(m_{\langle-1\rangle}\right) a_{0} \otimes \cdots \otimes a_{n}\right)=\varphi(m \otimes \widetilde{a})$.

Here the action of $H$ on $A^{\otimes(n+1)}$ is diagonal. We use the symbol ${ }_{A} H-\mathcal{S} \mathcal{A} \mathcal{Y D}$ for the category whose objects are ${ }_{A} H$-SAYD modules and whose morphisms are $H$-module and $H$-comodule morphisms

Lemma 2.16. Let $A$ be an $H$-module algebra. Then any SAYD module over $H$ is an ${ }_{A} H$-SAYD module.

Proof. One easily checks that the following properties are satisfied: i) $m_{\langle 0\rangle} S^{-1}\left(m_{\langle-1\rangle}\right)=m$, ii) $\varphi(m \otimes h \widetilde{a})=\varphi(m h \otimes \widetilde{a})$ if and only if $\varphi \in C_{H}^{*}(A, M)$, i.e. $\varphi\left(m h^{(1)} \otimes S\left(h^{(2)}\right) \widetilde{a}\right)=\varepsilon(h) \varphi(m \otimes \widetilde{a})$.

Definition 2.17. Let $A$ be an algebra and an $H$-module (not necessarily an $H$-module algebra). A module-comodule $M$ over $H$ is called an ${ }_{A} H$-Hopf cyclic coefficients $\left({ }_{A} H\right.$-HCC), if the cosimplicial and cyclic operators on $C_{H}^{*}(A, M)$ are well-defined and turn it into a cocyclic module. Here the action of $H$ on $A^{\otimes(n+1)}$ is diagonal. We use the symbol $A H$ - $\mathcal{H C C}$ for the category whose objects are ${ }_{A} H$-HCC modules and whose morphisms are $H$-module and $H$-comodule morphisms.

Proposition 2.18. Let $A$ be a left $H$-module algebra. Then any ${ }_{A} H$-SAYD module is an ${ }_{A} H$ HCC.

Proof. Let $M$ be a right-left ${ }_{A} H$-SAYD module. To show that $M$ is ${ }_{A} H$-HCC it is enough to check that the cyclic map is well-defined

$$
\begin{aligned}
& \left(t_{n} f\right)\left(\left(m \otimes a_{0} \otimes \cdots \otimes a_{n}\right) h\right)=\left(t_{n} f\right)\left(m h^{(1)} \otimes S\left(h^{(2)}\right)\left(a_{0} \otimes \cdots \otimes a_{n}\right)\right) \\
& =\left(t_{n} f\right)\left(m h^{(1)} \otimes S\left(h^{(n+2)}\right) a_{0} \otimes \cdots \otimes S\left(h^{(2)}\right) a_{n}\right) \\
& =f\left(\left(m h^{(1)}\right)_{\langle 0\rangle} \otimes S^{-1}\left(\left(m h^{(1)}\right)_{\langle-1\rangle}\right) S\left(h^{(2)}\right) a_{n} \otimes S\left(h^{(n+3)}\right) a_{0} \otimes \cdots \otimes S\left(h^{(3)}\right) a_{n-1}\right) \\
& =f\left(m_{\langle 0\rangle} h^{(2)} \otimes S^{-1}\left(S\left(h^{(3)}\right) m_{\langle-1\rangle} h^{(1)}\right) S\left(h^{(4)}\right) a_{n} \otimes S\left(h^{(n+4)}\right) a_{0} \otimes \cdots \otimes S\left(h^{(5)}\right) a_{n-1}\right) \\
& =f\left(m_{\langle 0\rangle} h^{(2)} \otimes S^{-1}\left(h^{(1)}\right) S^{-1}\left(m_{\langle-1\rangle}\right) h^{(3)} S\left(h^{(4)}\right) a_{n} \otimes S\left(h^{(n+4)}\right) a_{0} \otimes \cdots \otimes S\left(h^{(5)}\right) a_{n-1}\right) \\
& =f\left(m_{\langle 0\rangle} \otimes h^{(2)}\left[S^{-1}\left(h^{(1)}\right) S^{-1}\left(m_{\langle-1\rangle}\right) a_{n} \otimes S\left(h^{(n+2)}\right) a_{0} \otimes \cdots \otimes S\left(h^{(3)}\right) a_{n-1}\right]\right) \\
& =f\left(m_{\langle 0\rangle} \otimes h^{(2)} S^{-1}\left(h^{(1)}\right) S^{-1}\left(m_{\langle-1\rangle}\right) a_{n} \otimes h^{(3)} S\left(h^{(2 n+2)}\right) a_{0} \otimes \cdots \otimes h^{(n+2)} S\left(h^{(n+3)}\right) a_{n-1}\right) \\
& =f\left(m_{\langle 0\rangle} \otimes S^{-1}\left(m_{\langle-1\rangle}\right) a_{n} \otimes a_{0} \otimes \cdots \otimes a_{n-1}\right) \varepsilon(h) \\
& =\left(t_{n} f\right)\left(m \otimes a_{0} \otimes \cdots \otimes a_{n}\right) \varepsilon(h)=\left(\left(t_{n} f\right)\left(m \otimes a_{0} \otimes \cdots \otimes a_{n}\right)\right) h .
\end{aligned}
$$

To prove the cyclicity, using the ${ }_{A} H$-stability condition we have

$$
\begin{aligned}
& \left(t_{n}{ }^{n+1} f\right)\left(m \otimes a_{0} \otimes \cdots \otimes a_{n}\right)=\left(t_{n} f\right)\left(m_{\langle 0\rangle} \otimes S^{-1}\left(m_{\langle-1\rangle}\right) a_{1} \otimes \cdots \otimes a_{n} \otimes a_{0}\right) \\
& =\left(t_{n} f\right)\left(m \otimes a_{1} \otimes \cdots \otimes a_{n} \otimes a_{0}\right)=f\left(m \otimes a_{0} \otimes \cdots \otimes a_{n}\right) .
\end{aligned}
$$

Lemma 2.19. Let $A$ be a left $H$-module algebra, and $(\delta, \sigma)$ be a modular pair and ${ }_{A} H$-in involution, i.e.

$$
S_{\delta}^{-2}(h) a=\sigma^{-1} h \sigma a, \quad \text { for any } a \in A, \quad h \in H .
$$

Then ${ }^{\sigma} \mathbb{C}_{\delta}$ is an ${ }_{A} H$-SAYD module. 
Proof. The following computations show the ${ }_{A} H$-AYD condition

$$
\begin{aligned}
& S^{-1}\left(\left(1_{A} h\right)_{\langle-1\rangle}\right) a \otimes\left(1_{A} h\right)_{\langle 0\rangle}=\delta(h) S^{-1}(\sigma) a \otimes 1=\sigma^{-1} \delta\left(h^{(1)}\right) S\left(h^{(2)}\right) h^{(3)} a \otimes 1 \\
& \quad=\sigma^{-1} S_{\delta}\left(h^{(1)}\right) \sigma \sigma^{-1} h^{(2)} a \otimes 1=S_{\delta}^{-2}\left(S_{\delta}\left(h^{(1)}\right)\right) \sigma^{-1} h^{(2)} a \otimes 1 \\
& \quad=S^{-1}\left(h^{(1)}\right) \sigma^{-1} h^{(3)} a \otimes \delta\left(h^{(2)}\right)=S^{-1}\left(1_{\langle-1\rangle} h^{(1)}\right) h^{(3)} a \otimes 1_{\langle 0\rangle} h^{(2)} .
\end{aligned}
$$

The stability condition is obvious from the modular pair condition.

Proposition 2.20. Let $(\delta, \sigma)$ be a modular pair for $H$ and $A$ be a left $H$-module algebra. We define the following subspace of $A$

$$
B=\left\{a \in A \mid S_{\delta}^{-2}(h) a=\sigma^{-1} h \sigma a, \text { for all } h \in H\right\} .
$$

Then $B$ is an $H$-module subalgebra of $A$ and $(\delta, \sigma)$ is a ${ }_{B} H$-modular pair in involution.

Proof. It is obvious that $1 \in B$. Since $A$ is an $H$-module algebra, $\sigma$ is a group-like element and $S_{\delta}^{-2}$ is a coalgebra map, the following computation shows that $B$ is a subalgebra of $A$

$$
S_{\delta}^{-2}(h)(a b)=\left(S_{\delta}^{-2}\left(h^{(1)}\right) a\right)\left(S_{\delta}^{-2}\left(h^{(2)}\right) b\right)=\left(\sigma^{-1} h^{(1)} \sigma a\right)\left(\sigma^{-1} h^{(2)} \sigma b\right)=\sigma^{-1} h \sigma(a b) .
$$

Let $h \in H$ and $b \in B$. The following computation shows that $h b \in B$ and therefore that $B$ is a left $H$-module

$$
\begin{aligned}
\sigma^{-1} k \sigma(h b) & =\left(\sigma^{-1}\left(k \sigma h \sigma^{-1}\right) \sigma\right) b=S_{\delta}^{-2}\left(k \sigma h \sigma^{-1}\right) b \\
& =S_{\delta}^{-2}(k)\left[S_{\delta}^{-2}\left(\sigma h \sigma^{-1}\right) b\right]=S_{\delta}^{-2}(k) \sigma^{-1}\left(\sigma h \sigma^{-1}\right) \sigma b=S_{\delta}^{-2}(k)(h b) .
\end{aligned}
$$

Therefore $B$ is an $H$-module subalgebra of $A$ and $(\delta, \sigma)$ is a ${ }_{B} H$-modular pair in involution.

Lemma 2.21. Let $A$ be a left $H$-module algebra. If the action of $H$ on $A$ is cocommutative, i.e.

$$
h^{(1)} a_{1} \otimes h^{(2)} a_{2}=h^{(2)} a_{1} \otimes h^{(1)} a_{2}, \quad h \in H, \quad a_{1}, a_{2} \in A,
$$

then any module $M$ over $H$, with the trivial coaction of $H$, defines an ${ }_{A} H$-HCC.

Proof. The ${ }_{A} H$-stability condition is obvious by the triviality of the coaction. Since $A$ is assumed to be $H$-module algebra it suffices to show that the cyclic map $\tau$ is well-defined

$$
\begin{aligned}
\left(t_{n} f\right) & \left(\left(m \otimes a_{0} \otimes \cdots \otimes a_{n}\right) h\right)=\left(t_{n} f\right)\left(m h^{(1)} \otimes S\left(h^{(n+1)}\right) a_{0} \otimes \cdots \otimes S\left(h^{(2)}\right) a_{n}\right) \\
& =f\left(m h^{(1)} \otimes S\left(h^{(2)}\right) a_{n} \otimes S\left(h^{(n+1)}\right) a_{0} \otimes \cdots \otimes S\left(h^{(3)}\right) a_{n-1}\right) \\
& =f\left(m h^{(1)} \otimes S\left(h^{(n+1)}\right) a_{n} \otimes S\left(h^{(n)}\right) a_{0} \otimes \cdots \otimes S\left(h^{(2)}\right) a_{n-1}\right) \\
& =f\left(m \otimes a_{n} \otimes a_{0} \otimes \cdots \otimes a_{n-1}\right) \varepsilon(h)=\left(t_{n} f\right)\left(m \otimes a_{0} \otimes \cdots \otimes a_{n}\right) \varepsilon(h) .
\end{aligned}
$$

We use (2.9) in the third equality.

Lemma 2.22. Let $A$ be a left $H$-module algebra. If $H$ acts on $A$ commutatively, i.e.

$$
h g a=g h a, \quad h, g \in H, \quad a \in A,
$$

then any comodule $M$ over $H$, endowed with the trivial action from $H$, defines an ${ }_{A} H$-SAYD module. 
Proof. The ${ }_{A} H$-stability condition is obvious. The following computation proves the ${ }_{A} H$-AYD condition

$$
\begin{aligned}
& \varepsilon(h) S^{-1}\left(m_{\langle-1\rangle}\right) a \otimes m_{\langle 0\rangle}=h^{(2)} S^{-1}\left(h^{(1)}\right) S^{-1}\left(m_{\langle-1\rangle}\right) a \otimes m_{\langle 0\rangle} \\
& \quad=S^{-1}\left(m_{\langle-1\rangle} h^{(1)}\right) h^{(2)} a \otimes m_{\langle 0\rangle}=S^{-1}\left(m_{\langle-1\rangle} h^{(1)}\right) h^{(3)} a \otimes m_{\langle 0\rangle} h^{(2)} .
\end{aligned}
$$

We use the commutativity of the action on the second equality.

Here we introduce an example of the preceding lemma.

Example 2.23. Similar to Example 2.9, let $G$ be a group acting normally on a set $X$ from the right and $H=\mathbb{C} G$ be the group algebra of $G$ acting on $A=\operatorname{Fun}(X, \mathbb{C})$, the commutative algebra of all complex valued functions on $X$, by $(g f)(x)=f(x g)$. It is easy to check that $A$ is a left $H$-module algebra and furthermore that this action is commutative.

One notes that for any bicrossed product Hopf algebra $H=\mathcal{F} \bowtie \mathcal{U}$, the Hopf algebra $\mathcal{F}$ is an $H$-module algebra by the following action

$$
(f \bowtie u) g:=\varepsilon(f)(u g), \quad f, g \in \mathcal{F}, \quad u \in \mathcal{U} .
$$

Example 2.24. Let $H:=\mathcal{F} \bowtie \mathcal{U}$ be any bicrossed product Hopf algebra where $\mathcal{U}$ is a cocommutative Hopf algebra. Then the following computation proves that the action in (2.10) is cocommutative

$$
\begin{aligned}
& (f \bowtie u)^{(1)} v_{1} \otimes(f \bowtie u)^{(2)} v_{2}=\left(f^{(1)} \bowtie u_{\langle 0\rangle}^{(1)}\right) v_{1} \otimes\left(f^{(2)} u_{\langle 1\rangle}^{(1)} \bowtie u^{(2)}\right) v_{2} \\
& =\varepsilon\left(f^{(1)}\right) u_{\langle 0\rangle}^{(1)} v_{1} \otimes \varepsilon\left(f^{(2)} u_{\langle 1\rangle}^{(1)}\right) u^{(2)} v_{2}=\varepsilon(f) u^{(1)} v_{1} \otimes u^{(2)} v_{2}=(f \bowtie u)^{(2)} v_{1} \otimes(f \bowtie u)^{(1)} v_{2} .
\end{aligned}
$$

Therefore any module $M$ over $H$, with the trivial coaction of $H$, defines a $\mathcal{F} H$-HCC.

Here we introduce an example which shows that the categories of ${ }_{A} H$-HCC and ${ }_{A} H$-SAYD modules are different. In fact we introduce an example of an ${ }_{A} H$-HCC module which is not a ${ }_{A} H$-SAYD.

Example 2.25. Consider $\mathcal{H}=\mathcal{H}_{1}^{\text {cop }} \cong \mathcal{F} \bowtie \mathcal{U}$ and let $M=\mathcal{H}$ be a right $\mathcal{H}$-module via multiplication and left comodule by trivial coaction. We have seen that the Hopf algebra $\mathcal{F}$ is a left $\mathcal{H}$-module algebra by the action defined in (2.10) and this action is a cocommutative action by Example 2.24 and therefore that $M$ is a $\mathcal{F} \mathcal{H}$-HCC module. We show that $M$ is not a $\mathcal{F} \mathcal{H}$-AYD. First one notices that using the formula

$$
S^{-1}(h)=\delta\left(S\left(h^{(3)}\right)\right) \delta\left(h^{(1)}\right) S\left(h^{(2)}\right),
$$

we obtain

$$
\begin{aligned}
& S^{-1}\left(\delta_{1} \bowtie 1\right)=-\delta_{1} \bowtie 1, \quad S^{-1}\left(\delta_{2} \bowtie 1\right)=-\delta_{2} \bowtie 1, \\
& S^{-1}\left(\delta_{1} \bowtie Y\right)=\delta_{1} \bowtie Y+\delta_{1} \bowtie 1, \quad S^{-1}(1 \bowtie Y)=-1 \bowtie Y, \\
& S^{-1}(1 \bowtie X)=-(1 \bowtie X)+\left(\delta_{1} \bowtie Y\right), \\
& S^{-1}\left(\delta_{1}^{2} \bowtie Y\right)=-S^{-1}\left(2 \delta_{1}^{2} \bowtie 1\right)-\left(\delta_{1}^{2} \bowtie Y\right), \\
& S^{-1}\left(\delta_{1} \bowtie X\right)=\left(\delta_{1} \bowtie X\right)-\left(\delta_{2} \bowtie 1\right)-\left(\delta_{1}^{2} \bowtie Y\right) .
\end{aligned}
$$

Using (2.7) and the triviality of the coaction, by substituting $h=\delta_{1} \bowtie X, m=1 \bowtie 1$ and $a=\delta_{1}$ into the ${ }_{F} \mathcal{H}$-AYD condition we have

$$
S^{-1}\left(\left(\delta_{1} \bowtie X\right)^{(1)}\right)\left(\delta_{1} \bowtie X\right)^{(3)} \delta_{1} \otimes m\left(\delta_{1} \bowtie X\right)^{(2)}
$$




$$
\begin{aligned}
= & S^{-1}\left(\delta_{1}^{(1)} X_{\langle 0\rangle}^{(1)}\right)\left(\delta_{1}^{(3)} X_{\langle 2\rangle}^{(1)} X_{\langle 1\rangle}^{(2)} \bowtie X^{(3)}\right) \delta_{1} \otimes(1 \bowtie 1)\left(\delta_{1}^{(2)} X_{\langle 1\rangle}^{(1)} \bowtie X_{\langle 0\rangle}^{(2)}\right) \\
= & S^{-1}\left(\delta_{1}^{(1)} \bowtie X_{\langle 0\rangle}^{(1)}\right) X^{(3)} \delta_{1} \otimes\left(\delta_{1}^{(2)} X_{\langle 1\rangle}^{(1)} \bowtie X^{(2)}\right) \\
= & S^{-1}\left(\delta_{1}^{(1)} \bowtie X_{\langle 0\rangle}\right) \delta_{1} \otimes\left(\delta_{1}^{(2)} X_{\langle 1\rangle} \bowtie 1\right)+S^{-1}\left(\delta_{1}^{(1)} \bowtie 1\right) \delta_{1} \otimes\left(\delta_{1}^{(2)} \bowtie X\right) \\
& +S^{-1}\left(\delta_{1}^{(1)} \bowtie 1\right) X \delta_{1} \otimes\left(\delta_{1}^{(2)} \bowtie 1\right) \\
= & S^{-1}\left(\delta_{1}^{(1)} \bowtie X\right) \delta_{1} \otimes\left(\delta_{1}^{(2)} \bowtie 1\right)+S^{-1}\left(\delta_{1}^{(1)} \bowtie Y\right) \delta_{1} \otimes\left(\delta_{1}^{(2)} \delta_{1} \bowtie 1\right) \\
& +S^{-1}\left(\delta_{1}^{(1)} \bowtie 1\right) \delta_{1} \otimes\left(\delta_{1}^{(2)} \bowtie X\right)+S^{-1}\left(\delta_{1}^{(1)} \bowtie 1\right) X \delta_{1} \otimes\left(\delta_{1}^{(2)} \bowtie 1\right) \\
= & S^{-1}\left(\delta_{1} \bowtie X\right) \delta_{1} \otimes(1 \bowtie 1)+S^{-1}(1 \bowtie X) \delta_{1} \otimes\left(\delta_{1} \bowtie 1\right) \\
& +S^{-1}\left(\delta_{1} \bowtie Y\right) \delta_{1} \otimes\left(1 \delta_{1} \bowtie 1\right)+S^{-1}(1 \bowtie Y) \delta_{1} \otimes\left(\delta_{1} \delta_{1} \bowtie 1\right) \\
& +S^{-1}\left(\delta_{1} \bowtie 1\right) \delta_{1} \otimes(1 \bowtie X)+S^{-1}(1 \bowtie 1) \delta_{1} \otimes\left(\delta_{1} \bowtie X\right) \\
& +S^{-1}\left(\delta_{1} \bowtie 1\right) X \delta_{1} \otimes(1 \bowtie 1)+S^{-1}(1 \bowtie 1) X \delta_{1} \otimes\left(\delta_{1} \bowtie 1\right) \\
= & \left(\left(\delta_{1} \bowtie X\right)-\left(\delta_{2} \bowtie 1\right)-\left(\delta_{1}^{2} \bowtie Y\right)\right) \delta_{1} \otimes(1 \bowtie 1)+\left(-(1 \bowtie X)+\left(\delta_{1} \bowtie Y\right)\right) \delta_{1} \\
& \otimes\left(\delta_{1} \bowtie 1\right)+\left(\left(\delta_{1} \bowtie Y\right)+\delta_{1} \bowtie 1\right) \delta_{1} \otimes\left(\delta_{1} \bowtie 1\right)-(1 \bowtie Y) \delta_{1} \otimes\left(\delta_{1}^{2} \bowtie 1\right) \\
& -\left(\delta_{1} \bowtie 1\right) \delta_{1} \otimes(1 \bowtie X)+(1 \bowtie 1) \delta_{1} \otimes\left(\delta_{1} \bowtie X\right) \\
& -\left(\delta_{1} \bowtie 1\right) X \delta_{1} \otimes(1 \bowtie 1)+(1 \bowtie 1) X \delta_{1} \otimes\left(\delta_{1} \bowtie 1\right) \\
= & -\left(X \delta_{1}\right) \otimes\left(\delta_{1} \bowtie 1\right)-\left(Y \delta_{1}\right) \otimes\left(\delta_{1}^{2} \bowtie 1\right)+\delta_{1} \otimes\left(\delta_{1} \bowtie X\right)+\left(X \delta_{1}\right) \otimes\left(\delta_{1} \bowtie 1\right) \\
= & \delta_{1} \otimes\left(\delta_{1} \bowtie X\right)-\delta_{1} \otimes\left(\delta_{1}^{2} \bowtie 1\right) \\
\neq & \delta_{1} \otimes\left(\delta_{1} \bowtie X\right)=\delta_{1} \otimes(1 \bowtie 1)\left(\delta_{1} \bowtie X\right) .
\end{aligned}
$$

\section{Cup products in Hopf cyclic cohomology}

In this section we show that all features of Hopf cyclic cohomology work well with the new coefficients which we have defined in Section 2.1. One of the most important features of Hopf cyclic cohomology is its cup product $[9,10,14]$ as a generalization of the Connes-Moscovici characteristic map [4]. We show that this cup product works well with the new coefficients in this paper. One may construct an AYD module over a Hopf algebra as the tensor product of a YD module with a AYD module $[6,16]$. This shows that the category of AYD modules is a $\mathcal{C}$-category over the category of YD-modules. In this section we show the same expectation for generalized modules is satisfied. In other words we prove that the category of ${ }_{C} H$-SAYD modules is a $\mathcal{C}$-category over the category of ${ }_{C} H$-YD modules.

Definition 3.1. Let $C$ be a left $H$-module coalgebra. A left-right module-comodule $M$ over $H$ is called an $C H$-YD module if for $m \in M, h \in H$, and $c \in C$ we have

$$
(m h)_{\langle-1\rangle} c \otimes(m h)_{\langle 0\rangle}=S^{-1}\left(h^{(3)}\right) m_{\langle-1\rangle} h^{(1)} c \otimes m_{\langle 0\rangle} h^{(2)} .
$$

Lemma 3.2. Let $M$ and $N$ be right-left ${ }_{C} H$-anti-Yetter-Drinfeld and Yetter-Drinfeld module respectively. Then $M \otimes N$ is an ${ }_{C} H$-anti-Yetter-Drinfeld module via

$$
(m \otimes n) h=m h^{(2)} \otimes n h^{(1)}, \quad m \otimes n \longmapsto m_{\langle-1\rangle} n_{\langle-1\rangle} \otimes m_{\langle 0\rangle} \otimes n_{\langle-1\rangle} .
$$

Proof. The following computation shows that $M \otimes N$ is an ${ }_{C} H$-anti-Yetter-Drinfeld module

$$
\begin{aligned}
((m & \otimes n) h)_{\langle-1\rangle} c \otimes((m \otimes n) h)_{\langle 0\rangle}=\left(m h^{(2)} \otimes n h^{(1)}\right)_{\langle-1\rangle} c \otimes\left(m h^{(2)} \otimes n h^{(1)}\right)_{\langle 0\rangle} \\
& =\left(m h^{(2)}\right)_{\langle-1\rangle}\left(n h^{(1)}\right)_{\langle-1\rangle} c \otimes\left(m h^{(2)}\right)_{\langle 0\rangle} \otimes\left(n h^{(1)}\right)_{\langle 0\rangle} \\
& =S\left(h^{(6)}\right) m_{\langle-1\rangle} h^{(4)} S^{-1}\left(h^{(3)}\right) n_{\langle-1\rangle} h^{(1)} c \otimes m_{\langle 0\rangle} h^{(5)} \otimes n_{\langle 0\rangle} h^{(2)}
\end{aligned}
$$




$$
\begin{aligned}
& =S\left(h^{(4)}\right) m_{\langle-1\rangle} n_{\langle-1\rangle} h^{(1)} \otimes m_{\langle 0\rangle} h^{(3)} \otimes n_{\langle 0\rangle} h^{(2)} \\
& =S\left(h^{(3)}\right)(m \otimes n)_{\langle-1\rangle} h^{(1)} \otimes(m \otimes n)_{\langle 0\rangle} h^{(2)} .
\end{aligned}
$$

The rest of this section is devoted to the cup product in Hopf cyclic cohomology with generalized coefficients.

Let $C$ be an $H$-left module coalgebra acting from left on an $H$-left module algebra $A$ satisfying the following conditions

$$
(h c) a=h(c a), \quad c(a b)=\left(c^{(1)} a\right)\left(c^{(2)} b\right), \quad c 1=\varepsilon(c) 1 .
$$

Let $B=\operatorname{Hom}_{H}(C, A)$ denotes the convolution algebra of all $H$-linear maps from $C$ to $A$ with the following multiplication

$$
(f * g)(c)=f\left(c^{(1)}\right) g\left(c^{(2)}\right) .
$$

This algebra has the unit element $\eta \circ \varepsilon$ where $\eta: \mathbb{C} \longrightarrow A$ is the unit of $A$. Let $C_{H}^{*}(C, M)$ and $C_{H}^{*}(A, M)$ be the cocyclic modules which are defined in (2.1) and (2.8) where $M$ 's are right-left ${ }_{C} H$ and ${ }_{A} H$-SAYD modules respectively. Consider the diagonal complex

$$
C_{a, c}^{n, n}=C_{H}^{n}(C, M) \otimes C_{H}^{n}(A, M)=\operatorname{Hom}_{H}\left(M \otimes A^{\otimes n+1}, \mathbb{C}\right) \otimes\left(M \otimes_{H} C^{\otimes(n+1)}\right),
$$

which is a cocyclic module by the following coface, codegeneracy and cyclic maps, $\left(d_{n} \otimes \delta_{n}, s_{n} \otimes\right.$ $\left.\sigma_{n}, t_{n} \otimes \tau_{n}\right)$. For all $\varphi \in \operatorname{Hom}_{H}\left(M \otimes A^{\otimes n+1}, \mathbb{C}\right), f_{i} \in B$ and $m \otimes \widetilde{c} \in M \otimes_{H} C^{\otimes(n+1)}$, we define the following map

$$
\begin{aligned}
\Psi_{a, c}: & C_{a, c}^{n, n} \longrightarrow \operatorname{Hom}\left(B^{\otimes(n+1)}, \mathbb{C}\right), \\
& \Psi_{a, c}\left(\varphi \otimes m \otimes c_{0} \otimes \cdots \otimes c_{n}\right)\left(f_{0} \otimes \cdots \otimes f_{n}\right)=\varphi\left(m \otimes_{H} f_{0}\left(c_{0}\right) \otimes \cdots \otimes f_{n}\left(c_{n}\right)\right) .
\end{aligned}
$$

Similar to [14] one has the following statement.

Lemma 3.3. The map $\Psi_{a, c}$ is a well-defined map of cocyclic modules $C^{*, *}$ and $C^{*}(B)$.

Using (3.1), one shows that the following map is an unital algebra map

$$
\begin{gathered}
\chi: A \longrightarrow \operatorname{Hom}_{H}(C, A), \\
\chi(a)(c)=c(a) .
\end{gathered}
$$

Therefore we obtain a cyclic map $\chi: C^{*}(B, \mathbb{C}) \longrightarrow C^{*}(A, \mathbb{C})$. As a result we have the following cyclic map

$$
\Psi=\chi \circ \Psi_{a, c}: C_{a, c}^{n, n} \longrightarrow C^{n}(A, \mathbb{C}) .
$$

Now we define

$$
C_{a, c}^{p, q}=\bigoplus_{p+q=n} C_{H}^{p}(A, M) \otimes C_{H}^{q}(C, M)
$$

to be a tensor product of cocyclic modules which has a mixed complex structure $[2,12]$. The cyclic cohomology of this mixed complex is

$$
\bigoplus_{p, q} H C_{H}^{p}(A, M) \otimes H C_{H}^{q}(C, M)
$$

As in [14], one uses the Alexander-Whitney map to obtain the following map

$$
\mathrm{AW}: H C_{H}^{p}(A, M) \otimes H C_{H}^{q}(C, M) \longrightarrow H C^{p+q}\left(C_{a, c}^{p, q}\right) \longrightarrow H C^{n}\left(C_{a, c}^{n, n}\right) .
$$

Now we obtain the following statement. 
Proposition 3.4. Let $C$ be an $H$-left module coalgebra acting from the left on an $H$-left module algebra $A$ satisfying (3.1). Let $M$ be an ${ }_{A} H$ and ${ }_{C} H$-SAYD module, and let $C_{H}^{*}(C, M)$ and $C_{H}^{*}(A, M)$ be the cocyclic modules which are defined in (2.1) and (2.8). The following maps define cup products on the level of Hopf cyclic cohomology

$$
\sqcup=\Psi \circ \mathrm{AW}: H C_{H}^{p}(A, M) \otimes H C_{H}^{q}(C, M) \longrightarrow H C^{p+q}(A) .
$$

Proof. This can be proved similarly as the author did in [14].

\section{Acknowledgements}

The authors of the manuscript are thankful to the organizers of Focus Program on Noncommutative Geometry and Quantum Groups, which was held at Fields Institute June 3-28, 2013 for the invitation and the support. Special thanks to P.M. Hajac for his valuable comments and his unique attention to Hopf cyclic cohomology. Last but not least, we would like to thank the referees for their extremely helpful comments. This work is part of the project supported by the NCN grant 2011/01/B/ST1/06474.

\section{References}

[1] Böhm G., Ştefan D., (Co)cyclic (co)homology of bialgebroids: an approach via (co)monads, Comm. Math. Phys. 282 (2008), 239-286, arXiv:0705.3190.

[2] Connes A., Cohomologie cyclique et foncteurs $\mathrm{Ext}^{n}$, C. R. Acad. Sci. Paris Sér. I Math. 296 (1983), 953-958.

[3] Connes A., Noncommutative geometry, Academic Press, Inc., San Diego, CA, 1994.

[4] Connes A., Moscovici H., Hopf algebras, cyclic cohomology and the transverse index theorem, Comm. Math. Phys. 198 (1998), 199-246, math.DG/9806109.

[5] Hajac P.M., Khalkhali M., Rangipour B., Sommerhäuser Y., Hopf-cyclic homology and cohomology with coefficients, C. R. Math. Acad. Sci. Paris 338 (2004), 667-672, math.KT/0306288.

[6] Hajac P.M., Khalkhali M., Rangipour B., Sommerhäuser Y., Stable anti-Yetter-Drinfeld modules, C. R. Math. Acad. Sci. Paris 338 (2004), 587-590, math.QA/0405005.

[7] Jara P., Ştefan D., Hopf-cyclic homology and relative cyclic homology of Hopf-Galois extensions, Proc. London Math. Soc. 93 (2006), 138-174, math.KT/0307099.

[8] Kaygun A., Bialgebra cyclic homology with coefficients, K-Theory 34 (2005), 151-194, math.KT/0409191.

[9] Kaygun A., Products in Hopf-cyclic cohomology, Homology, Homotopy Appl. 10 (2008), 115-133, arXiv:0710.2559.

[10] Khalkhali M., Rangipour B., Cup products in Hopf-cyclic cohomology, C. R. Math. Acad. Sci. Paris 340 (2005), 9-14, math.QA/0411003.

[11] Kowalzig N., Krähmer U., Cyclic structures in algebraic (co)homology theories, Homology Homotopy Appl. 13 (2011), 297-318, arXiv:1011.3471.

[12] Loday J.L., Cyclic homology, Grundlehren der Mathematischen Wissenschaften, Vol. 301, Springer-Verlag, Berlin, 1992.

[13] Moscovici H., Rangipour B., Hopf algebras of primitive Lie pseudogroups and Hopf cyclic cohomology, Adv. Math. 220 (2009), 706-790, arXiv:0803.1320.

[14] Rangipour B., Cup products in Hopf cyclic cohomology via cyclic modules, Homology, Homotopy Appl. 10 (2008), 273-286, arXiv:0710.2623.

[15] Rangipour B., Sütlü S., SAYD modules over Lie-Hopf algebras, Comm. Math. Phys. 316 (2012), 199-236, arXiv:1108.6101.

[16] Staic M.D., A note on anti-Yetter-Drinfeld modules, in Hopf algebras and generalizations, Contemp. Math., Vol. 441, Amer. Math. Soc., Providence, RI, 2007, 149-153. 